Pensamiento Crítico Vol. 18 Nº 1, pp. 43-55

\title{
Diversificación de la Cartera de Activos de la Banca Múltiple en el Perú: 2001-2011
}

Gaby Cortez Cortez

\section{RESUMEN}

La diversificación de las operaciones de los bancos en el mayor número de sectores económicos, todavía tiene un espacio asignado por la Teoría Financiera Clásica, que sugiere que la diversificación puede ser una manera de reducir el riesgo de una cartera de activos (Haugen, 2001) asociada a beneficios más altos y menores costos de operación. Durante el periodo 2001-2011 los bancos que desarrollaron sus actividades en el mercado bancario del Perú han mostrado un alto grado de diversificación expresado en la participación en un número mayoritario de sectores económicos, también asociado a mayores tasas de retorno del capital expresado a través del ratio $\mathrm{ROE}$ (Return on Equity).

Palabras clave: diversificación, activos bancarios, rentabilidad

\section{ABSTRACT}

Diversification of bank operations in the most economic sectors, still has space allocated for Classical Financial Theory, which suggests that diversification may be a way to reduce the risk of a portfolio of assets (Haugen, 2001) associated 


\section{Pensamiento Crítico Vol. I8. Nº}

with higher profits and lower operating costs. During the period 2001-2011 the banks that are active in the banking market in Peru have shown a high degree of diversification expressed in the majority stake in a number of economic sectors, also associated with higher rates of return on capital expressed through ratio $\mathrm{ROE}$ (Return on Equity)

Keywords: diversification, bank assets, profitability.

\section{INTRODUCCIÓN}

¿Qué es la diversificación en una institución financiera? Algunos autores sostienen que diversificar es colocar los activos de un banco en una amplia gama de prestatarios de calidad, para mantener ó mejorar los niveles de ganancias mientras que se mantiene el mismo nivel de exposición.

También se señala que la diversificación puede ser vista como la oferta de productos y servicios por parte de un banco. Asimismo, puede verse la diversificación desde la perspectiva de las fuentes de ingresos, por sectores económicos, por industrias, o por regiones. Debe tenerse en cuenta que hay beneficios y riesgos en la diversificación de los activos de las instituciones financieras, especialmente en instituciones que trabajan con recursos del público tal como en el caso de los bancos.

La teoría clásica de las finanzas sugiere que una mayor diversificación en la cartera de préstamos de un banco debe reducir el riesgo realizado, medido por el importe de las provisiones para préstamos incobrables (hipótesis de la diversificación clásica).

También se argumenta que si la gerencia carece del tiempo ó de la experiencia suficiente para controlar o supervisar efectivamente el proceso de concesión de préstamos a nuevos segmentos de clientes o a nuevas industrias, una mayor diversificación no necesariamente implica menos provisiones (hipótesis de falta de pericia, o de expertise).

Una segunda razón porque la alta diversificación no se traduce necesariamente en una menor necesidad de provisiones puede ser debido a la calidad y composición de la cartera. Si un banco tiene una cartera menos diversificada, concentrada en actividades de bajo riesgo de crédito, la necesidad de constituir provisiones aún podría ser inferior a 


\section{Gaby Cortez Cortez}

la de un banco con una cartera más diversificada de activos de alto riesgo. Por lo tanto, se tiene que controlar la calidad de la cartera de un banco cuando se analiza el impacto de la diversificación del riesgo.

\section{Revisión de la literatura}

La literatura sobre la diversificación en la banca puede ser abordada desde diferentes perspectivas, tal como a continuación se presenta:

La investigación de Stiroh, Kevin, Diversification in Banking: Is Noninterest Income the Answer? (2004), en donde se evalúa los posibles beneficios de la diversificación en la industria bancaria en los Estados Unidos, como resultado del constante traslado de sus operaciones hacia los ingresos no financieros. Se sostiene que a nivel agregado, la disminución de la volatilidad de los ingresos netos operativos refleja la disminución de la volatilidad de los ingresos netos por intereses, y no debido a los beneficios de la diversificación de los ingresos que no provienen de intereses, que son bastante volátiles y cada vez mas correlacionados con los ingresos netos por intereses. También se argumenta que a nivel de los bancos, una mayor dependencia de los ingresos no provenientes de intereses, en particular los ingresos por trading, se asocian con un menor beneficio ajustado por el riesgo y por un mayor riesgo. Esto sugiere pocos beneficios provenientes de la diversificación a partir del desplazamiento continuo hacia Ingresos no provenientes de intereses.

En el caso particular del sistema bancario peruano se ha observado que solamente durante los dos últimos años del periodo bajo análisis se ha experimentado un aumento importante de este tipo de operaciones que incluyen a los ingresos no financieros. Es así que el crédito indirecto / PBI ha tenido una participación de 3.83 por ciento del 2001 al 2009, mientras que del 2010 al 2011 el promedio pasa a 18.5 por ciento, lo que indica que los bancos recién han incursionado en este tipo de operaciones de manera más activa a fin de diversificar sus activos, por lo tanto es prematuro dar respuestas sobre la perspectivas de desarrollo en esta línea por parte de los bancos.

En el trabajo de Saunders, Anthony y Acharya, Viral.,titulado Should Banks Be Diversified? Evidence from Individual Bank Loan Portfolios (2006), se estudia el efecto del enfoque de la concentración de la cartera de préstamos versus la diversificación sobre 


\section{Pensamiento Crítico Vol. I8. Nº}

la rentabilidad y el riesgo de 105 bancos italianos durante el periodo 1993-1999, para lo cual se usaron datos de los bancos en las distintas industrias y sectores. Los autores encuentran que la diversificación no es una garantía para obtener un desempeño superior y/o una mayor seguridad para los bancos. Para aquellos bancos que incurrieron en alto riesgo, la diversificación disminuyó la rentabilidad al mismo tiempo que se produjeron préstamos riesgosos. Para el caso de los bancos con riesgo bajo, la diversificación generó ya sea una compensación ineficiente entre riesgo y rentabilidad o sólo una mejora marginal. Los resultados a los que llegaron los autores son consistentes con el deterioro en la efectividad de la supervisión de los bancos con altos niveles de riesgo, y generando posteriormente la expansión de los préstamos hacia industrias nuevas y competitivas.

Al respecto, si bien los resultados de las regresiones efectuadas en el presente trabajo para los diversos bancos del Perú muestran cierta coherencia con la teoría, incluir el riesgo podría mejorar el enfoque respecto a los planteamientos ortodoxos de la teoría clásica de la diversificación. Considerando que el modelo que se ha aplicado para cada banco muestra en sus resultados coeficientes de correlación bajos en algunos casos, los que a su vez están asociados a errores estándar altos, nos indicaría que la diversificación si bien es un elemento importante, no es determinante para la explicación de la rentabilidad asociada a ésta.

En la investigación The Anatomy of Bank Diversification de Elsas, Ralf., Hackenthal, Andreas (2010), se comprueba para nueve países durante el periodo 1996-2008, cómo afecta la diversificación de los ingresos al valor de los bancos. Los autores se basan en un marco global para la medición del desempeño de los bancos. Se sostiene que la diversificación aumenta la rentabilidad de los bancos y como consecuencia el valor de mercado de los mismos. Este efecto de performance indirecto no depende de si la diversificación se logra a través de un crecimiento orgánico o a través de fusiones y adquisiciones. Además, demuestran que los trabajos similares previos sobre el impacto de la diversificación en el valor de un banco, se presume que difieren debido a la forma en que se mide la diversificación.

En el caso de los bancos peruanos se ha encontrado que la diversificación afecta de manera positiva a la rentabilidad de los bancos, sin embargo, esta confirmación de la teoría no ha sido contrastada con el riesgo inherente que líneas arriba se ha mencionado, lo cual sería deseable para completar la robustez de la teoría. 


\section{Gaby Cortez Cortez}

En el trabajo de Stiroh, Kevin The dark side of diversification: The case of US financial holding companies (2006), se plantea que los beneficios potenciales de la diversificación son una de las razones por las cuales los holding financieros de los Estados Unidos están ofreciendo una gama creciente de servicios financieros. Los expertos analizan si el cambio hacia las actividades que generan comisiones, ingresos por trading, $y$ otros ingresos distintos de los intereses han permitido mejorar el rendimiento de los holdings financieros de las empresas estadounidenses desde 1997 hasta el 2002. El autor encuentra evidencia de que existen beneficios de la diversificación entre los holding financieros, pero estos beneficios se contrarrestan con el incremento de la exposición de las actividades que no generan intereses, las que son mucho más volátiles, pero no necesariamente más rentables que las actividades que generan intereses. El hallazgo principal es que los beneficios de la diversificación son más que contrarrestados por los costos de una mayor exposición de las actividades volátiles, lo que representa el lado oscuro de la búsqueda de beneficios a través de la diversificación y que tiene implicancias significativas para los entes supervisores, gerentes, inversionistas y prestatarios.

Para el caso de los cuatro bancos grandes que incrementaron su participación en actividades no financieras durante los años 2010 y 2011 se requiere de un seguimiento en los años venideros a fin de poder evaluar su efecto en la rentabilidad de los bancos, debido a que la lectura de las cifras no nos permite sacar conclusiones definitivas respecto a la importancia de estas nuevas operaciones en la rentabilidad de dichas empresas, ya que la misma ha venido disminuyendo a partir del 2009, luego de un pico extraordinario de ROE promedio de 78.6 por ciento en el 2008. En contraste los créditos indirectos han tenido una fuerte subida dentro del total de créditos otorgados en los años 2010 y 2011, pasando de un promedio de 16.7 por ciento del 2001 al 2009 a 41.7 por ciento del 2010 al 2011.

En el trabajo de Rossi, Stefania y Schwaiger, Markus, How loan portfolio diversification affects risk, efficiency and capitalization: A managerial behavior model for Austrian banks (2009), se indica que el objetivo de la investigación es analizar cómo la diversificación de los bancos de todo tamaño e industria afecta el riesgo, el costo, la eficiencia del beneficio, y la capitalización bancaria de los grandes bancos comerciales austriacos durante los años 1997-2003. Para cumplir su objetivo emplean un conjunto de datos proporcionados por el Banco Central de Austria y ponen a prueba varias hipótesis, para lo cual emplean el modelo de Berger y DeYoung. Los autores encuentran 


\section{Pensamiento Crítico Vol. I8. Nº}

que a pesar que la diversificación afecta negativamente a la eficiencia del costo, aumenta la eficiencia de la rentabilidad, y disminuye el riesgo bancario. Finalmente sostienen que la diversificación parece tener un impacto positivo en la capitalización bancaria.

Este trabajo es importante porque amplía la visión o aplicabilidad de la diversificación asociada a un conjunto mayor de variables que podrían enriquecer aun más el tema y desarrollarse en trabajos posteriores.

\section{Problema}

¿Por qué los bancos diversifican sus activos?

¿Por qué es importante el estudio de la diversificación de los activos de los bancos?

Los bancos en la búsqueda de la diversificación de sus activos, a fin de elevar sus beneficios, pueden afectar de manera directa a los consumidores en la medida que la diversificación de sus activos los puede llevar a elevar sus costos, a generar ineficiencias, y a disminuir el bienestar de los que hacen uso de sus servicios y de los que no pueden tener acceso a los mismos. En atención a estos planteamientos los entes reguladores deben vigilar el comportamiento de la diversificación de los activos de los bancos.

\section{Hipótesis}

Hipótesis general: "La diversificación de la cartera de activos tiene un efecto positivo sobre el rendimiento de los bancos en el Perú en el periodo 2001-2011".

Hipótesis secundaria 1: "La diversificación reduce el riesgo medido por el monto de provisiones constituidas por los bancos para malos préstamos"

Hipótesis secundaria 2: "La diversificación disminuye los costos y aumenta la eficiencia para la obtención de beneficios".

\section{Prueba de hipótesis}

Un instrumento importante en la medición de la concentración de los activos de los bancos es el Índice Herfindhal - Hirshman ( $\mathrm{IHH}$ ), que señala que cuando éste se acerca más a la unidad corresponderá a un mayor nivel de concentración de activos. En sentido 


\section{Gaby Cortez Cortez}

contrario, cuanto más alejado de la unidad se encuentre este índice, se considera que habrá una menor concentración y por lo tanto una mayor diversificación. Se ha utilizado para la elaboración del $\mathrm{IHH}$ los créditos directos, de consumo e hipotecarios para los nueve bancos del sistema que se mantuvieron funcionando durante todo el periodo de análisis tal como se muestra en el Cuadro 1. El IHH promedio de estos bancos es de 0.193 para el periodo de análisis y el nivel promedio correspondientes a los cuatro bancos principales del sistema fue de 0.139 , indicándonos la mayor diversificación de estas últimas instituciones.

\section{Cuadro 1 Indice H-H de Créditos Directos, de Consumo e}

Hipotecarios de Bancos Grandes: 2001-2011

\section{(En Porcentaje)}

\begin{tabular}{|c|c|c|c|c|c|}
\hline Año & 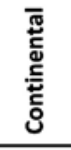 & 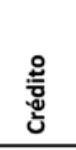 & 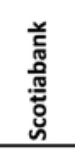 & 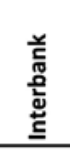 & 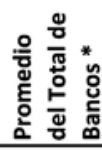 \\
\hline 2001 & 0.134 & 0.140 & 0.123 & 0.120 & 0.171 \\
\hline 2002 & 0.131 & 0.141 & 0.120 & 0.122 & 0.166 \\
\hline 2003 & 0.140 & 0.161 & 0.117 & 0.136 & 0.172 \\
\hline 2004 & 0.136 & 0.141 & 0.118 & 0.172 & 0.189 \\
\hline 2005 & 0.128 & 0.140 & 0.122 & 0.174 & 0.196 \\
\hline 2006 & 0.131 & 0.133 & 0.118 & 0.190 & 0.188 \\
\hline 2007 & 0.136 & 0.129 & 0.118 & 0.180 & 0.201 \\
\hline 2008 & 0.127 & 0.124 & 0.108 & 0.181 & 0.211 \\
\hline 2009 & 0.121 & 0.118 & 0.106 & 0.184 & 0.212 \\
\hline 2010 & 0.127 & 0.117 & 0.104 & 0.175 & 0.211 \\
\hline 2011 & 0.124 & 0.118 & 0.099 & 0.178 & 0.208 \\
\hline Prom. & 0.130 & 0.133 & 0.128 & 0.165 & 0.193 \\
\hline D.E. & 0.063 & 0.013 & 0.044 & 0.026 & \\
\hline
\end{tabular}

El nivel promedio del $\mathrm{IHH}$ de los nueve bancos se ha incrementado de manera importante desde 0.171 en el 2001 hasta 0.208 en el 2011, mientras que el nivel del IHH de tres de los cuatro bancos grandes del sistema ha disminuido a lo largo del periodo de análisis, haciéndose notar que en el caso de Interbank muestra una mayor concentración que los otros tres bancos. Se debe señalar que Scotiabank ha tenido un mejor manejo de la diversificación al mostrar un $\mathrm{IHH}$ menor que el correspondiente a los bancos de Crédito y Continental. 


\section{Pensamiento Crítico Vol. 18. $\mathrm{N}^{\circ}$ I}

De otro lado, el IHH promedio del resto de los bancos que no se han mantenido durante todo el periodo de análisis ha sido de 0.414 tal como se aprecia en el Cuadro 2 . Este nivel es mucho más alto que el correspondiente a los bancos con mayores activos. Se puede observar que el grado de concentración aumenta de manera importante para el caso de los bancos Falabella (0.961) y Ripley (0.986), ya que ambos tienen concentrados sus créditos a través de la venta al retail.

Asimismo, el IHH promedio de este segundo grupo de bancos pequeños es superior al de los bancos grandes o de mayores activos, indicándonos de esta manera que los bancos grandes manejan mejor la diversificación de la cartera de activos. (Ver el Gráfico 1).

Cuadro 2

H-H de Créditos Directos, de Consumo e Hipotecarios por Bancos Pequeños

(En Porcentaje)

\begin{tabular}{|c|c|c|c|c|c|c|c|c|c|c|}
\hline Año & $\begin{array}{l}\text {. } \\
\text { 포 }\end{array}$ & 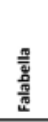 & 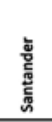 & 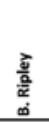 & $\begin{array}{l}\text { 을 } \\
\text { 률 }\end{array}$ & 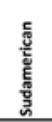 & 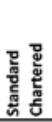 & 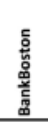 & 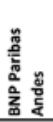 & 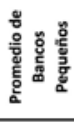 \\
\hline 2001 & & & 0.125 & & 0.431 & 0.202 & 0.287 & 0.205 & & 0.250 \\
\hline 2002 & & & 0.148 & & 0.424 & 0.321 & 0.239 & 0.204 & 0.340 & 0.279 \\
\hline 2003 & & & & & 0.405 & 0.254 & 0.277 & 0.290 & 0.318 & 0.309 \\
\hline 2004 & & & & & 0.386 & 0.196 & 0.284 & 0.242 & 0.277 & 0.277 \\
\hline 2005 & & & & & 0.365 & 0.156 & & & 0.565 & 0.362 \\
\hline 2006 & 0.244 & & & & 0.363 & & & & & 0.304 \\
\hline 2007 & 0.202 & 0.942 & 0.193 & & 0.371 & & & & & 0.427 \\
\hline 2008 & 0.176 & 0.954 & 0.285 & 0.953 & 0.357 & & & & & 0.545 \\
\hline 2009 & 0.158 & 0.959 & 0.280 & 0.992 & & & & & & 0.597 \\
\hline 2010 & 0.200 & 0.972 & 0.225 & 0.999 & & & & & & 0.599 \\
\hline 2011 & 0.181 & 0.980 & 0.257 & 0.999 & & & & & & 0.604 \\
\hline Prom. & 0.194 & 0.961 & 0.216 & 0.986 & 0.388 & 0.226 & 0.272 & 0.235 & 0.375 & 0.414 \\
\hline D.E. & 0.030 & 0.015 & 0.039 & 0.022 & 0.029 & 0.064 & 0.022 & 0.041 & 0.129 & \\
\hline
\end{tabular}

Gráfico 1 HH de Créditos Directos, de Consumo e Hipotecarios de Bancos:2001-2011

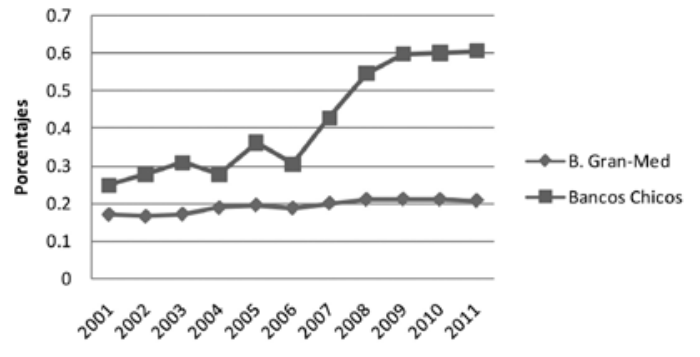




\section{Gaby Cortez Cortez}

\section{Hipótesis General}

Se plantea como hipótesis principal que "La diversificación de la cartera de activos tiene un efecto positivo sobre el rendimiento de los bancos en el Perú en el periodo 2001-2011". Para la prueba estadística de la hipótesis general se ha considerado la siguiente función:

$\mathrm{ROE}=\mathrm{f}(\mathrm{IHH}-$, MOR-, LGAC +$)$

Variable independiente: ROE

Variables dependientes: IHH, MOR, LGAC(*)

Los resultados de la regresión se muestran en el Cuadro 3 en donde se observa que la rentabilidad de los bancos guarda una relación directa con signo negativo respecto a la diversificación de sus activos, lo que se cumple para los seis bancos materia de analisis. Asimismo, se encuentra que la morosidad tiene un efecto negativo sobre la rentabilidad de los bancos, observándose un aumento de estos mayores costos para el caso de Mibanco e Interbank, en donde se tienen carteras de activos menos diversificadas.

Cuadro 3. ROE $=f(-I H H,-M O R, L G A C)$

\begin{tabular}{|lcccc|}
\hline & Variable & Coeficiente & Error Estándar & R2 \\
& & & & \\
\hline Banco Continental & IHH & -3.251441 & 2.166054 & 0.86801 \\
& MOR & -6.742855 & 1.549332 & \\
Banco de Crédito & IHH & -1.669473 & 3.447400 & 0.657438 \\
& MOR & -8.642243 & 3.457880 & \\
Interbank & IHH & -2.476465 & 1.625092 & 0.924484 \\
& MOR & -10.05568 & 1.803707 & \\
Mibanco & IHH & -0.642145 & 0.364909 & 0.790291 \\
& MOR & -12.82050 & 4.768574 & \\
B. Interamericano & IHH & -1.249708 & 1.173397 & 0.782838 \\
& MOR & -2.927697 & 1.143637 & \\
Scotiabank & IHH & -1.343872 & 7.492689 & 0.454898 \\
& MOR & -6.569397 & 4.340976 & \\
\hline
\end{tabular}

Fuente: Superintendencia de Banca y Seguros

Elaboración: Gaby Cortez

* IHH(Indice Herfendhal-Hirshman), MOR(Creditos Atrasados), LGAC (logaritmo Activo Total) 


\section{Pensamiento Crítico Vol. 18. $\mathrm{N}^{\circ}$ I}

\section{Hipótesis secundaria 1}

Se considera que "La diversificación reduce el riesgo medido por el monto de provisiones constituidas por los bancos para malos préstamos"

Para la prueba estadística de la hipótesis secundaria 1 se ha considerado la siguiente función:

$\mathrm{ROE}=\mathrm{f}(\mathrm{IHH}-$, PROV-, LGAC +$)$

Variable independiente: ROE

Variables dependientes: IHH, PROV, LGAC(**)

En este caso, también se prueba que la diversificación contribuye a la rentabilidad de los bancos, lo que se observa a través de los signos negativos de los coeficientes del IHH tal como se muestra en el cuadro 4. También se observa que los signos negativos de los coeficientes de las provisiones nos muestran que tienen un efecto adverso sobre la rentabilidad al constituir un costo importante para los bancos, lo cual disminuye los beneficios de estas empresas financieras y que corresponde con los resultados obtenidos.

Cuadro 4. ROE $=f(-$ IHH, - PROV, LGAC +$)$

\begin{tabular}{|lllll|}
\hline & Variable & Coeficiente & Error Estándar & R2 \\
& & & & \\
\hline Banco Continental & IHH & -0.907702 & 0.658931 & 0.76681 \\
& PROV & -10.37961 & 3.154689 & \\
Banco de Crédito & IHH & -1.629281 & 3.908245 & 0.70290 \\
& PROV & -10.69758 & 3.685199 & \\
Interbank & IHH & -2.704320 & 1.997751 & 0.89861 \\
& PROV & -8.242156 & 1.793970 & \\
Mibanco & IHH & -0.720235 & 0.267123 & 0.869702 \\
& PROV & -8.900502 & 2.190546 & \\
B. Interamericano & IHH & -0.215021 & 0.682348 & 0.933051 \\
& PROV & -2.946103 & 0.470505 & \\
Scotiabank & IHH & -4.393312 & 14.06076 & 0.450196 \\
& PROV & -10.97291 & 5.820678 & \\
\hline
\end{tabular}

Fuente: Superintendencia de Banca y Seguros

Elaboración: Gaby Cortez

** IHH(Indice Herfindhal-Hirshman), PROV(Provisiones), LGAC(Logaritmo Activo Total) 


\section{Gaby Cortez Cortez}

\section{Hipótesis Secundaria 2}

La hipótesis secundaria 2 sostiene que "La diversificación disminuye los costos y aumenta la eficiencia para la obtención de beneficios". Para la prueba estadística de la hipótesis secundaria 2 se ha considerado la siguiente función:

$\mathrm{ROE}=\mathrm{f}(\mathrm{IHH}-, \mathrm{CO}-, \mathrm{LGAC}+)$

Variable independiente: $\mathrm{ROE}$

Variables dependientes: IHH, CO, LGAC(***)

Cuadro 5.ROE $=f($ IHH-, CO-, LGAC+ $)$

\begin{tabular}{|lcccc|}
\hline & Variable & Coeficiente & Error Estándar & R2 \\
\hline Banco Continental & IHH & -2.632514 & 0.510773 & 0.8346 \\
Banco de Crédito & CO & -29.97835 & 6.961846 & \\
& IHH & -5.848144 & 4.758297 & 0.40202 \\
Interbank & $\mathrm{CO}$ & -4.107708 & 10.226840 & \\
& $\mathrm{IHH}$ & -6.518184 & 2.379405 & 0.6452 \\
Mibanco & $\mathrm{CO}$ & -2.987380 & 5.290440 & \\
& $\mathrm{IHH}$ & -1.264996 & 0.746868 & 0.6022 \\
B. Interamericano & $\mathrm{CO}$ & -2.375905 & 2.825536 & \\
& $\mathrm{IHH}$ & -3.054454 & 1.136088 & 0.6370 \\
Scotiabank & $\mathrm{CO}$ & -2.375905 & 2.825536 & \\
& $\mathrm{IHH}$ & -16.65718 & 12.93727 & 0.2107 \\
\hline
\end{tabular}

Fuente: Superintendencia de Banca y Seguros Elaboración: Gaby Cortez

Los resultados que se han conseguido indican que la relación entre la diversificación y los costos es significativa, de tal manera que ante mayores costos incurridos por los bancos, la rentabilidad se verá afectada negativamente.

\section{CONCLUSIONES}

El mercado bancario en el Perú muestra un grado significativo de diversificación de sus operaciones en el conjunto de sectores económicos del país durante el periodo 2001-2011.

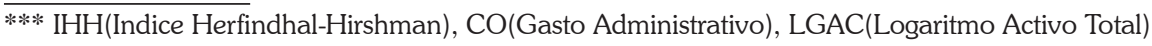




\section{Pensamiento Crítico Vol. I8. N I}

El volumen de las operaciones de los bancos y su presencia en un mayor número de sectores económicos, determina su capacidad competitiva a través de la obtención de mayores beneficios expresados por tasas de retorno del capital por encima del promedio de mercado.

El sistema bancario en conjunto durante el periodo 2001-2011 muestra un grado de diversificación promedio medido a través del Índice Herfindhal - Hirshman de 0.27, por debajo del índice máximo de concentración que es 1 , señalándonos el avance general en la diversificación.

Los bancos grandes y medianos muestran un índice $\mathrm{HH}$ de 0.193 en promedio durante el periodo de análisis, mientras que los bancos pequeños muestran un índice $\mathrm{HH}$ de 0.414 por encima del promedio de los bancos del sistema, mostrándonos una mayor diversificación de los bancos grandes respecto a los bancos pequeños.

En términos generales los resultados empíricos confirman a través de una muestra de bancos, que existe una relación entre la rentabilidad de los bancos y la diversificación de sus operaciones, asociadas a variables de riesgo y escala de operaciones que se puede resumir en el siguiente ranking.

\section{Indicadores Promedio de Bancos}

Seleccionados: 2001-2011

\begin{tabular}{lcc}
\hline Bancos & ROE & IHH \\
\hline Crédito & 45.1 & 0.133 \\
Continental & 44.2 & 0.130 \\
Interbank & 37.2 & 0.165 \\
Scotiabank & 36.3 & 0.128 \\
Interamericano & 15.0 & 0.150 \\
Financiero & 5.8 & 0.150 \\
Mibanco & 42.2 & 0.307 \\
\hline Total Banca & 33.7 & 0.193 \\
\hline
\end{tabular}

Fuente: SBS

Elaboración: Gaby Cortez 


\section{Gaby Cortez Cortez}

\section{Comentario final}

En la banca, sin embargo, la diversificación de por sí no es garantía de una reducción en el riesgo de fracaso o para lograr un mejor desempeño. Una razón para esto es la importancia que tiene la supervisión de préstamos o la calidad de la cartera.

\section{BIBLIOGRAFÍA}

Estadísticas del Banco Central de Reserva del Perú

Estadísticas de la Superintendencia de Banca y Seguros del Perú

Elsas, Ralf, Hackethal, Andreas, Holzhauser, Markus. The Anatomy of Bank Diversification (2010). Journal of Banking \& Finance.

Rossi, Stefania., Schwaiger, Markus., Winkler, Gerhard. How loan portfolio diversification affects risk, efficiency and capitalization: A managerial behavior model for Austrian banks (2009): Journal of Banking \& Finance.

Saunders, Anthony., Acharya, Viral., Hasan, Iftekhar. Should Banks Be Diversified? Evidence from Individual Bank Loan Portfolios (2006). Journal of Business.

Stiroh, Kevin. Diversification in Banking: Is Noninterest Income the Answer? (2004). Journal of Money, Credit and Banking.

Stiroh, Kevin, Rumble, Adrienne. The dark side of diversification: The case of US financial holding companies (2006). Journal of Banking \& Finance. 